\title{
Cytotoxic Effects of Phlomis Brevibracteata Turril. on SK-HEP-1 Adenocarcinoma Cell Line ${ }^{\dagger}$
}

\author{
Imge Kunter, Beste Atli *, Ezgi Ak, Nesrin Oztinen, Andia Babri and Muberra Kosar \\ Faculty of Pharmacy, Eastern Mediterranean University, North Cyprus via Mersin 10, \\ 99628 Famagusta, Turkey; imge.kunter@emu.edu.tr (I.K.); ezgiiakk@hotmail.com (E.A.); \\ nesrinnesrinoztinen@gmail.com (N.O.); andiababri72@gmail.com (A.B.); muberra.kosar@emu.edu.tr (M.K.) \\ * Correspondence: beste.atli@gmail.com; Tel.: +90-392-630-3110 \\ + Presented at the 3rd International conference on Natural Products for Cancer Prevention and Therapy, \\ Kayseri, Turkey, 18-20 December 2019.
}

Published: 27 December 2019

\begin{abstract}
Phlomis is a large genus in the Lamiaceae family with over 100 species distributed throughout Europe, Asia and North Africa. Some species of Phlomis are used in folk medicine as a stimulant, tonic, and for wound healing. Many studies have shown that different Phlomis species have anti-inflammatory, immunosuppressive, free radical scavenging, antimicrobial, antiulcerogenic and anti-mutagenic activities. Among these species, Phlomis brevibracteata, Turrill, is an endemic plant which grows in Kyrenia, Karpasia, Nicosia and Limassol regions of Cyprus. In our previous studies, we found that methanol extract (PBM) has anti-carcinogenic activity, in terms of cytotoxicity and 2D motility inhibition. In this study, we tried to find out potential anti-carcinogenic activity of hexane (PBH), ethyl acetate (PBE) and butanol (PBB) sub-crude extracts of the methanol-extracts of the same plant. PBH, PBE and PBB extracts of herbal parts of the plant were used in different concentrations to assess the anti-carcinogenic activity on liver adenocarcinoma cell SK-HEP-1. MTT and wound healing assays were carried out to determine anti-cancinogenic activities. Our findings showed that all three extracts have negative effects on cell viability and motility with different levels. Among these extracts, PBE and PBH extracts were more potent than PBB in terms of IC 50 values.
\end{abstract}

Keywords: Phlomis brevibracteata; SK-Hep-1 cells; anti-carcinogenic activity

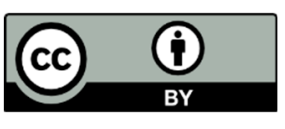

(C) 2019 by the authors. Licensee MDPI, Basel, Switzerland. This article is an open access article distributed under the terms and conditions of the Creative Commons Attribution (CC BY) license (http://creativecommons.org/licenses/by/4.0/). 